\title{
Public-Key Cryptosystems Based on Composite Degree Residuosity Classes
}

\author{
Pascal Paillier ${ }^{1,2}$ \\ 1 GEMPLUS \\ Cryptography Department \\ 34 Rue Guynemer, 92447 Issy-Les-Moulineaux \\ paillier@gemplus.com \\ 2 ENST \\ Computer Science Department \\ 46, rue Barrault, 75634 Paris Cedex 13 \\ paillier@inf.enst.fr
}

\begin{abstract}
This paper investigates a novel computational problem, namely the Composite Residuosity Class Problem, and its applications to public-key cryptography. We propose a new trapdoor mechanism and derive from this technique three encryption schemes : a trapdoor permutation and two homomorphic probabilistic encryption schemes computationally comparable to RSA. Our cryptosystems, based on usual modular arithmetics, are provably secure under appropriate assumptions in the standard model.
\end{abstract}

\section{Background}

Since the discovery of public-key cryptography by Diffie and Hellman [5], very few convincingly secure asymetric schemes have been discovered despite considerable research efforts.

We refer the reader to [26] for a thorough survey of existing public-key cryptosystems. Basically, two major species of trapdoor techniques are in use today. The first points to RSA [25] and related variants such as Rabin-Williams 24/30, LUC, Dickson's scheme or elliptic curve versions of RSA like KMOV [10]. The technique conjugates the polynomial-time extraction of roots of polynomials over a finite field with the intractability of factoring large numbers. It is worthwhile pointing out that among cryptosystems belonging to this family, only RabinWilliams has been proven equivalent to the factoring problem so far.

Another famous technique, related to Diffie-Hellman-type schemes (El Gamal [7], DSA, McCurley [14], etc.) combines the homomorphic properties of the modular exponentiation and the intractability of extracting discrete logarithms over finite groups. Again, equivalence with the primitive computational problem remains open in general, unless particular circumstances are reached as described in [12].

Other proposed mechanisms generally suffer from inefficiency, inherent security weaknesses or insufficient public scrutiny : McEliece's cryptosystem [15] 
based on error correcting codes, Ajtai-Dwork's scheme based on lattice problems (cryptanalyzed by Nguyen and Stern in [18]), additive and multiplicative knapsack-type systems including Merkle-Hellman [13, Chor-Rivest (broken by Vaudenay in [29]) and Naccache-Stern [17] ; finally, Matsumoto-Imai and Goubin-Patarin cryptosystems, based on multivariate polynomials, were successively cryptanalyzed in [11] and 21.

We believe, however, that the cryptographic research had unnoticeably witnessed the progressive emergence of a third class of trapdoor techniques : firstly identified as trapdoors in the discrete log, they actually arise from the common algebraic setting of high degree residuosity classes. After Goldwasser-Micali's scheme 9] based on quadratic residuosity, Benaloh's homomorphic encryption function, originally designed for electronic voting and relying on prime residuosity, prefigured the first attempt to exploit the plain resources of this theory. Later, Naccache and Stern [16, and independently Okamoto and Uchiyama [19] significantly extended the encryption rate by investigating two different approaches : residuosity of smooth degree in $\mathbb{Z}_{p q}^{*}$ and residuosity of prime degree $p$ in $\mathbb{Z}_{p^{2} q}^{*}$ respectively. In the meantime, other schemes like Vanstone-Zuccherato [28] on elliptic curves or Park-Won 20] explored the use of high degree residues in other settings.

In this paper, we propose a new trapdoor mechanism belonging to this family. By contrast to prime residuosity, our technique is based on composite residuosity classes i.e. of degree set to a hard-to-factor number $n=p q$ where $p$ and $q$ are two large prime numbers. Easy to understand, we believe that our trapdoor provides a new cryptographic building-block for conceiving public-key cryptosystems.

In sections 2 and 3, we introduce our number-theoretic framework and investigate in this context a new computational problem (the Composite Residuosity Class Problem), which intractability will be our main assumption. Further, we derive three homomorphic encryption schemes based on this problem, including a new trapdoor permutation. Probabilistic schemes will be proven semantically secure under appropriate intractability assumptions. All our polynomial reductions are simple and stand in the standard model.

Notations. We set $n=p q$ where $p$ and $q$ are large primes : as usual, we will denote by $\phi(n)$ Euler's totient function and by $\lambda(n)$ Carmichael's function 11 taken on $n$, i.e. $\phi(n)=(p-1)(q-1)$ and $\lambda(n)=\operatorname{lcm}(p-1, q-1)$ in the present case. Recall that $\left|\mathbb{Z}_{n^{2}}^{*}\right|=\phi\left(n^{2}\right)=n \phi(n)$ and that for any $w \in \mathbb{Z}_{n^{2}}^{*}$,

$$
\left\{\begin{array}{l}
w^{\lambda}=1 \bmod n \\
w^{n \lambda}=1 \bmod n^{2},
\end{array}\right.
$$

which are due to Carmichael's theorem. We denote by $\operatorname{RSA}[n, e]$ the (conventionally thought intractable) problem of extracting $e$-th roots modulo $n$ where $n=p q$ is of unknown factorisation. The relation $P_{1} \Leftarrow P_{2}$ (resp. $\left.P_{1} \equiv P_{2}\right)$ will denote that the problem $P_{1}$ is polynomially reducible (resp. equivalent) to the problem $P_{2}$.

\footnotetext{
${ }^{1}$ we will adopt $\lambda$ instead of $\lambda(n)$ for visual comfort.
} 


\section{Deciding Composite Residuosity}

We begin by briefly introducing composite degree residues as a natural instance of higher degree residues, and give some basic related facts. The originality of our setting resides in using of a square number as modulus. As said before, $n=p q$ is the product of two large primes.

Definition 1. A number $z$ is said to be a $n$-th residue modulo $n^{2}$ if there exists a number $y \in \mathbb{Z}_{n^{2}}^{*}$ such that

$$
z=y^{n} \bmod n^{2}
$$

The set of $n$-th residues is a multiplicative subgroup of $\mathbb{Z}_{n^{2}}^{*}$ of order $\phi(n)$. Each $n$-th residue $z$ has exactly $n$ roots of degree $n$, among which exactly one is strictly smaller than $n$ (namely $\sqrt[n]{z} \bmod n$ ). The $n$-th roots of unity are the numbers of the form $(1+n)^{x}=1+x n \bmod n^{2}$.

The problem of deciding $n$-th residuosity, i.e. distinguishing $n$-th residues from non $n$-th residues will be denoted by $\mathrm{CR}[n]$. Observe that like the problems of deciding quadratic or higher degree residuosity, $\mathrm{CR}[n]$ is a random-selfreducible problem that is, all of its instances are polynomially equivalent. Each case is thus an average case and the problem is either uniformly intractable or uniformly polynomial. We refer to [1,8] for detailed references on random-selfreducibility and the cryptographic significance of this feature.

As for prime residuosity (cf. [3,16]), deciding $n$-th residuosity is believed to be computationally hard. Accordingly, we will assume that :

Conjecture 2. There exists no polynomial time distinguisher for $n$-th residues modulo $n^{2}$, i.e. $\mathrm{CR}[n]$ is intractable.

This intractability hypothesis will be refered to as the Decisional Composite Residuosity Assumption (DCRA) throughout this paper. Recall that due to the random-self-reducibility, the validity of the DCRA only depends on the choice of $n$.

\section{Computing Composite Residuosity Classes}

We now proceed to describe the number-theoretic framework underlying the cryptosystems introduced in sections 4, 5] and 6. Let $g$ be some element of $\mathbb{Z}_{n^{2}}^{*}$ and denote by $\mathcal{E}_{g}$ the integer-valued function defined by

$$
\begin{aligned}
\mathbb{Z}_{n} \times \mathbb{Z}_{n}^{*} \longmapsto & \mathbb{Z}_{n^{2}}^{*} \\
(x, y) & \longmapsto g^{x} \cdot y^{n} \bmod n^{2}
\end{aligned}
$$

Depending on $g, \mathcal{E}_{g}$ may feature some interesting properties. More specifically,

Lemma 3. If the order of $g$ is a nonzero multiple of $n$ then $\mathcal{E}_{g}$ is bijective. 
We denote by $\mathcal{B}_{\alpha} \subset \mathbb{Z}_{n^{2}}^{*}$ the set of elements of order $n \alpha$ and by $\mathcal{B}$ their disjoint union for $\alpha=1, \cdots, \lambda$.

Proof. Since the two groups $\mathbb{Z}_{n} \times \mathbb{Z}_{n}^{*}$ and $\mathbb{Z}_{n^{2}}^{*}$ have the same number of elements $n \phi(n)$, we just have to prove that $\mathcal{E}_{g}$ is injective. Suppose that $g^{x_{1}} y_{1}^{n}=$ $g^{x_{2}} y_{2}^{n} \bmod n^{2}$. It comes $g^{x_{2}-x_{1}} \cdot\left(y_{2} / y_{1}\right)^{n}=1 \bmod n^{2}$, which implies $g^{\lambda\left(x_{2}-x_{1}\right)}=$ $1 \bmod n^{2}$. Thus $\lambda\left(x_{2}-x_{1}\right)$ is a multiple of $g$ 's order, and then a multiple of $n$. Since $\operatorname{gcd}(\lambda, n)=1, x_{2}-x_{1}$ is necessarily a multiple of $n$. Consequently, $x_{2}-x_{1}=0 \bmod n$ and $\left(y_{2} / y_{1}\right)^{n}=1 \bmod n^{2}$, which leads to the unique solution $y_{2} / y_{1}=1$ over $\mathbb{Z}_{n}^{*}$. This means that $x_{2}=x_{1}$ and $y_{2}=y_{1}$. Hence, $\mathcal{E}_{g}$ is bijective.

Definition 4. Assume that $g \in \mathcal{B}$. For $w \in \mathbb{Z}_{n^{2}}^{*}$, we call $n$-th residuosity class of $w$ with respect to $g$ the unique integer $x \in \mathbb{Z}_{n}$ for which there exists $y \in \mathbb{Z}_{n}^{*}$ such that

$$
\mathcal{E}_{g}(x, y)=w
$$

Adopting Benaloh's notations [3], the class of $w$ is denoted $\llbracket w \rrbracket_{g}$. It is worthwhile noticing the following property :

Lemma 5. $\llbracket w \rrbracket_{g}=0$ if and only if $w$ is a $n$-th residue modulo $n^{2}$. Furthermore,

$$
\forall w_{1}, w_{2} \in \mathbb{Z}_{n^{2}}^{*} \quad \llbracket w_{1} w_{2} \rrbracket_{g}=\llbracket w_{1} \rrbracket_{g}+\llbracket w_{2} \rrbracket_{g} \bmod n
$$

that is, the class function $w \mapsto \llbracket w \rrbracket_{g}$ is a homomorphism from $\left(\mathbb{Z}_{n^{2}}^{*}, \times\right)$ to $\left(\mathbb{Z}_{n},+\right)$ for any $g \in \mathcal{B}$.

The $n$-th Residuosity Class Problem of base $g$, denoted Class $[n, g]$, is defined as the problem of computing the class function in base $g$ : for a given $w \in \mathbb{Z}_{n^{2}}^{*}$, compute $\llbracket w \rrbracket_{g}$ from $w$. Before investigating further Class $[n, g]$ 's complexity, we begin by stating the following useful observations :

Lemma 6. Class $[n, g]$ is random-self-reducible over $w \in \mathbb{Z}_{n^{2}}^{*}$.

Proof. Indeed, we can easily transform any $w \in \mathbb{Z}_{n^{2}}^{*}$ into a random instance $w^{\prime} \in \mathbb{Z}_{n^{2}}^{*}$ with uniform distribution, by posing $w^{\prime}=w g^{\alpha} \beta^{n} \bmod n^{2}$ where $\alpha$ and $\beta$ are taken uniformly at random over $\mathbb{Z}_{n}$ (the event $\beta \notin \mathbb{Z}_{n}^{*}$ occurs with negligibly small probability). After $\llbracket w^{\prime} \rrbracket_{g}$ has been computed, one has simply to return $\llbracket w \rrbracket_{g}=\llbracket w^{\prime} \rrbracket_{g}-\alpha \bmod n$.

Lemma 7. Class $[n, g]$ is random-self-reducible over $g \in \mathcal{B}$, i.e.

$$
\forall g_{1}, g_{2} \in \mathcal{B} \quad \text { Class }\left[n, g_{1}\right] \equiv \text { Class }\left[n, g_{2}\right]
$$

Proof. It can easily be shown that, for any $w \in \mathbb{Z}_{n^{2}}^{*}$ and $g_{1}, g_{2} \in \mathcal{B}$, we have

$$
\llbracket w \rrbracket_{g_{1}}=\llbracket w \rrbracket_{g_{2}} \llbracket g_{2} \rrbracket_{g_{1}} \bmod n,
$$


which yields $\llbracket g_{1} \rrbracket_{g_{2}}=\llbracket g_{2} \rrbracket_{g_{1}}^{-1} \bmod n$ and thus $\llbracket g_{2} \rrbracket_{g_{1}}$ is invertible modulo $n$. Suppose that we are given an oracle for Class $\left[n, g_{1}\right]$. Feeding $g_{2}$ and $w$ into the oracle respectively gives $\llbracket g_{2} \rrbracket_{g_{1}}$ and $\llbracket w \rrbracket_{g_{1}}$, and by straightforward deduction :

$$
\llbracket w \rrbracket_{g_{2}}=\llbracket w \rrbracket_{g_{1}} \llbracket g_{2} \rrbracket_{g_{1}}^{-1} \bmod n .
$$

Lemma [7] essentially means that the complexity of Class $[n, g]$ is independant from $g$. This enables us to look upon it as a computational problem which purely relies on $n$. Formally,

Definition 8. We call Composite Residuosity Class Problem the computational problem Class $[n]$ defined as follows : given $w \in \mathbb{Z}_{n^{2}}^{*}$ and $g \in \mathcal{B}$, compute $\llbracket w \rrbracket_{g}$.

We now proceed to find out which connections exist between the Composite Residuosity Class Problem and standard number-theoretic problems. We state first :

Theorem 9. Class $[n] \Leftarrow \operatorname{Fact}[n]$.

Before proving the theorem, observe that the set

$$
\mathcal{S}_{n}=\left\{u<n^{2} \mid u=1 \bmod n\right\}
$$

is a multiplicative subgroup of integers modulo $n^{2}$ over which the function $\mathrm{L}$ such that

$$
\forall u \in \mathcal{S}_{n} \quad \mathrm{~L}(u)=\frac{u-1}{n}
$$

is clearly well-defined.

Lemma 10. For any $w \in \mathbb{Z}_{n^{2}}^{*}, L\left(w^{\lambda} \bmod n^{2}\right)=\lambda \llbracket w \rrbracket_{1+n} \bmod n$.

Proof (of Lemma 10). Since $1+n \in \mathcal{B}$, there exists a unique pair $(a, b)$ in the set $\mathbb{Z}_{n} \times \mathbb{Z}_{n}^{*}$ such that $w=(1+n)^{a} b^{n} \bmod n^{2}$. By definition, $a=\llbracket w \rrbracket_{1+n}$. Then

$$
w^{\lambda}=(1+n)^{a \lambda} b^{n \lambda}=(1+n)^{a \lambda}=1+a \lambda n \bmod n^{2},
$$

which yields the announced result.

Proof (of Theorem 9). Since $\llbracket g \rrbracket_{1+n}=\llbracket 1+n \rrbracket_{g}^{-1} \bmod n$ is invertible, a consequence of Lemma 10 is that $\mathrm{L}\left(g^{\lambda} \bmod n^{2}\right)$ is invertible modulo $n$. Now, factoring $n$ obviously leads to the knowledge of $\lambda$. Therefore, for any $g \in \mathcal{B}$ and $w \in \mathbb{Z}_{n^{2}}^{*}$, we can compute

$$
\frac{\mathrm{L}\left(w^{\lambda} \bmod n^{2}\right)}{\mathrm{L}\left(g^{\lambda} \bmod n^{2}\right)}=\frac{\lambda \llbracket w \rrbracket_{1+n}}{\lambda \llbracket g \rrbracket_{1+n}}=\frac{\llbracket w \rrbracket_{1+n}}{\llbracket g \rrbracket_{1+n}}=\llbracket w \rrbracket_{g} \bmod n,
$$

by virtue of Equation!1. 
Theorem 11. Class $[n] \Leftarrow R S A[n, n]$.

Proof. Since all the instances of Class $[n, g]$ are computationally equivalent for $g \in \mathcal{B}$, and since $1+n \in \mathcal{B}$, it suffices to show that

$$
\text { Class }[n, 1+n] \Leftarrow \operatorname{RSA}[n, n] .
$$

Let us be given an oracle for $\operatorname{RSA}[n, n]$. We know that $w=(1+n)^{x} \cdot y^{n} \bmod n^{2}$ for some $x \in \mathbb{Z}_{n}$ and $y \in \mathbb{Z}_{n}^{*}$. Therefore, we have $w=y^{n} \bmod n$ and we get $y$ by giving $w \bmod n$ to the oracle. From now,

$$
\frac{w}{y^{n}}=(1+n)^{x}=1+x n \bmod n^{2}
$$

which discloses $x=\llbracket w \rrbracket_{1+n}$ as announced.

Theorem 12. Let D-Class $[n]$ be the decisional problem associated to Class $[n]$ i.e. given $w \in \mathbb{Z}_{n^{2}}^{*}, g \in \mathcal{B}$ and $x \in \mathbb{Z}_{n}$, decide whether $x=\llbracket w \rrbracket_{g}$ or not. Then

$$
C R[n] \equiv D \text {-Class }[n] \Leftarrow \operatorname{Class}[n] .
$$

Proof. The hierarchy D-Class $[n] \Leftarrow$ Class $[n]$ comes from the general fact that it is easier to verify a solution than to compute it. Let us prove the left-side equivalence. $(\Rightarrow)$ Submit $w g^{-x} \bmod n^{2}$ to the oracle solving CR $[n]$. In case of $n$-th residuosity detection, the equality $\llbracket w g^{-x} \rrbracket_{g}=0$ implies $\llbracket w \rrbracket_{g}=x$ by Lemma [5] and then answer "Yes". Otherwise answer "No" or "Failure" according to the oracle's response. $(\Leftarrow)$ Choose an arbitrary $g \in \mathcal{B}(1+n$ will do) and submit the triple $(g, w, x=0)$ to the oracle solving D-Class $[n]$. Return the oracle's answer without change.

To conclude, the computational hierarchy we have been looking for was

$$
\mathrm{CR}[n] \equiv \operatorname{D}-\operatorname{Class}[n] \Leftarrow \operatorname{Class}[n] \Leftarrow \operatorname{RSA}[n, n] \Leftarrow \operatorname{Fact}[n]
$$

with serious doubts concerning a potential equivalence, excepted possibly between D-Class $[n]$ and Class $[n]$. Our second intractability hypothesis will be to assume the hardness of the Composite Residuosity Class Problem by making the following conjecture :

Conjecture 13. There exists no probabilistic polynomial time algorithm that solves the Composite Residuosity Class Problem, i.e. Class $[n]$ is intractable.

By contrast to the Decisional Composite Residuosity Assumption, this conjecture will be refered to as the Computational Composite Residuosity Assumption (CCRA). Here again, random-self-reducibility implies that the validity of the CCRA is only conditioned by the choice of $n$. Obviously, if the DCRA is true then the CCRA is true as well. The converse, however, still remains a challenging open question. 


\section{A New Probabilistic Encryption Scheme}

We now proceed to describe a public-key encryption scheme based on the Composite Residuosity Class Problem. Our methodology is quite natural : employing $\mathcal{E}_{g}$ for encryption and the polynomial reduction of Theorem 9 for decryption, using the factorisation as a trapdoor.

Set $n=p q$ and randomly select a base $g \in \mathcal{B}$ : as shown before, this can be done efficiently by checking whether

$$
\operatorname{gcd}\left(\mathrm{L}\left(g^{\lambda} \bmod n^{2}\right), n\right)=1 .
$$

Now, consider $(n, g)$ as public parameters whilst the pair $(p, q)$ (or equivalently $\lambda$ ) remains private. The cryptosystem is depicted below.

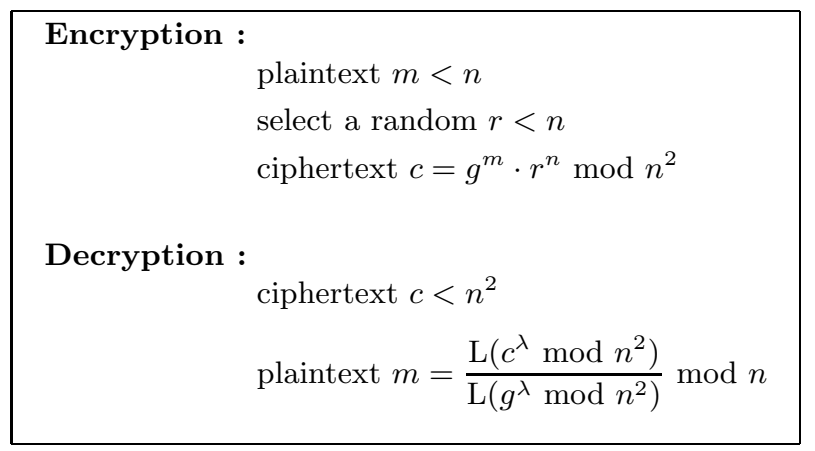

Scheme 1. Probabilistic Encryption Scheme Based on Composite Residuosity.

The correctness of the scheme is easily verified from Equation 2, and it is straightforward that the encryption function is a trapdoor function with $\lambda$ (that is, the knowledge of the factors of $n$ ) as the trapdoor secret. One-wayness is based on the computational problem discussed in the previous section.

Theorem 14. Scheme 1 is one-way if and only if the Computational Composite Residuosity Assumption holds.

Proof. Inverting our scheme is by definition the Composite Residuosity Class Problem.

Theorem 15. Scheme 1 is semantically secure if and only if the Decisional Composite Residuosity Assumption holds.

Proof. Assume that $m_{0}$ and $m_{1}$ are two known messages and $c$ the ciphertext of either $m_{0}$ or $m_{1}$. Due to Lemma 5, $c$ is the ciphertext of $m_{0}$ if and only if $c g^{-m_{0}} \bmod n^{2}$ is a $n$-th residue. Therefore, a successfull chosen-plaintext attacker could decide composite residuosity, and vice-versa. 


\section{A New One-Way Trapdoor Permutation}

One-way trapdoor permutations are very rare cryptographic objects : we refer the reader to [22] for an exhaustive documentation on these. In this section, we show how to use the trapdoor technique introduced in the previous section to derive a permutation over $\mathbb{Z}_{n^{2}}^{*}$.

As before, $n$ stands for the product of two large primes and $g$ is chosen as in Equation 4 .

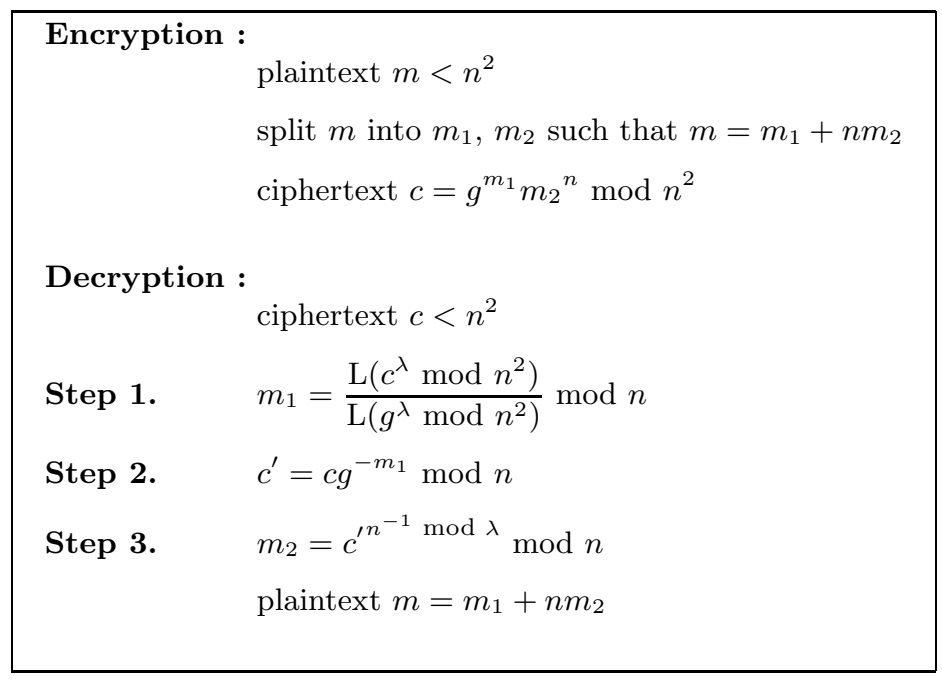

Scheme 2. A Trapdoor Permutation Based on Composite Residuosity.

We first show the scheme's correctness. Clearly, Step 1 correctly retrieves $m_{1}=m \bmod n$ as in Scheme 1 . Step 2 is actually an unblinding phase which is necessary to recover $m_{2}^{n} \bmod n$. Step 3 is an RSA decryption with a public exponent $e=n$. The final step recombines 2 the original message $m$. The fact that Scheme 2 is a permutation comes from the bijectivity of $\mathcal{E}_{g}$. Again, trapdoorness is based on the factorisation of $n$. Regarding one-wayness, we state :

Theorem 16. Scheme 2 is one-way if and only if $R S A[n, n]$ is hard.

Proof. a) Since Class $[n] \Leftarrow \operatorname{RSA}[n, n]$ (Theorem [11), extracting $n$-th roots modulo $n$ is sufficient to compute $m_{1}$ from $\mathcal{E}_{g}\left(m_{1}, m_{2}\right)$. Retrieving $m_{2}$ then requires one more additionnal extraction. Thus, inverting Scheme 2 cannot be harder than extracting $n$-th roots modulo $n$. b) Conversely, an oracle which inverts Scheme 2 allows root extraction : first query the oracle to get the two

$\overline{2}$ note that every public bijection $m \leftrightarrow\left(m_{1}, m_{2}\right)$ fits the scheme's structure, but euclidean division appears to be the most natural one. 
numbers $a$ and $b$ such that $1+n=g^{a} b^{n} \bmod n^{2}$. Now if $w=y_{0}^{n} \bmod n$, query the oracle again to obtain $x$ and $y$ such that $w=g^{x} y^{n} \bmod n^{2}$. Since $1+n \in \mathcal{B}$, we know there exists an $x_{0}$ such that $w=(1+n)^{x_{0}} y_{0}^{n} \bmod n^{2}$, wherefrom

$$
w=\left(g^{a} b^{n}\right)^{x_{0}} y_{0}^{n}=g^{a x_{0} \bmod n}\left(g^{a x_{0} \operatorname{div} n} b^{x_{0}} y_{0}\right)^{n} \bmod n^{2} .
$$

By identification with $w=g^{x} y^{n} \bmod n^{2}$, we get $x_{0}=x a^{-1} \bmod n$ and finally $y_{0}=y g^{-\left(a x_{0} \operatorname{div} n\right)} b^{-x_{0}} \bmod n$ which is the wanted value.

Remark 17. Note that by definition of $\mathcal{E}_{g}$, the cryptosystem requires that $m_{2} \in$ $\mathbb{Z}_{n}^{*}$, just like in the RSA setting. The case $m_{2} \notin \mathbb{Z}_{n}^{*}$ either allows to factor $n$ or leads to the ciphertext zero for all possible values of $m_{1}$. A consequence of this fact is that our trapdoor permutation cannot be employed ad hoc to encrypt short messages i.e. messages smaller than $n$.

Digital Signatures. Finally, denoting by $h: \mathbb{N} \mapsto\{0,1\}^{k} \subset \mathbb{Z}_{n^{2}}^{*}$ a hash function see as a random oracle 2], we obtain a digital signature scheme as follows. For a given message $m$, the signer computes the signature $\left(s_{1}, s_{2}\right)$ where

$$
\left\{\begin{array}{l}
s_{1}=\frac{\mathrm{L}\left(h(m)^{\lambda} \bmod n^{2}\right)}{\mathrm{L}\left(g^{\lambda} \bmod n^{2}\right)} \bmod n \\
s_{2}=\left(h(m) g^{-s_{1}}\right)^{1 / n \bmod \lambda} \bmod n
\end{array}\right.
$$

and the verifier checks that

$$
h(m) \stackrel{?}{=} g^{s_{1}} s_{2}^{n} \bmod n^{2} .
$$

Corollary 18 (of Theorem 16). In the random oracle model, an existential forgery of our signature scheme under an adaptive chosen message attack has a negligible success probability provided that $R S A[n, n]$ is intractable.

Although we feel that the above trapdoor permutation remains of moderate interest due to its equivalence with RSA, the rarity of such objects is such that we find it useful to mention its existence. Moreover, the homomorphic properties of this scheme, discussed in section 8 , could be of a certain utility regarding some (still unresolved) cryptographic problems.

\section{Reaching Almost-Quadratic Decryption Complexity}

Most popular public-key cryptosystems present a cubic decryption complexity, and this is the case for Scheme 1 as well. The fact that no faster (and still appropriately secure) designs have been proposed so far strongly motivates the search for novel trapdoor functions allowing increased decryption performances. This section introduces a slightly modified version of our main scheme (Scheme 1) which features an $\mathcal{O}\left(|n|^{2+\epsilon}\right)$ decryption complexity. 
Here, the idea consists in restricting the ciphertext space $\mathbb{Z}_{n^{2}}^{*}$ to the subgroup $\langle g\rangle$ of smaller order by taking advantage of the following extension of Equation 2 Assume that $g \in \mathcal{B}_{\alpha}$ for some $1 \leq \alpha \leq \lambda$. Then for any $w \in<g>$,

$$
\llbracket w \rrbracket_{g}=\frac{\mathrm{L}\left(w^{\alpha} \bmod n^{2}\right)}{\mathrm{L}\left(g^{\alpha} \bmod n^{2}\right)} \bmod n .
$$

This motivates the cryptosystem depicted below.

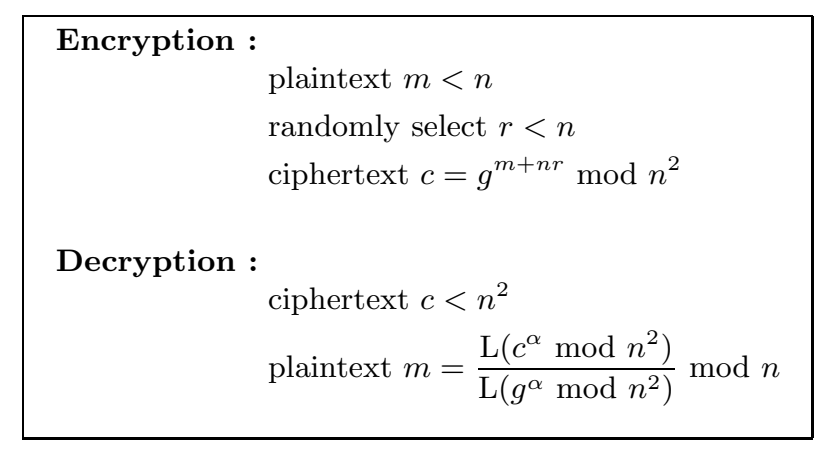

Scheme 3. Variant with fast decryption.

Note that this time, the encryption function's trapdoorness relies on the knowledge of $\alpha$ (instead of $\lambda$ ) as secret key. The most computationally expensive operation involved in decryption is the modular exponentiation $c \rightarrow c^{\alpha} \bmod n^{2}$ which runs in complexity $\mathcal{O}\left(|n|^{2}|\alpha|\right)$ (to be compared to $\mathcal{O}\left(|n|^{3}\right)$ in Scheme 1). If $g$ is chosen in such a way that $|\alpha|=\Omega\left(|n|^{\epsilon}\right)$ for some $\epsilon>0$, then decryption will only take $\mathcal{O}\left(|n|^{2+\epsilon}\right)$ bit operations. To the best of our knowledge, Scheme 3 is the only public-key cryptosystem based on modular arithmetics whose decryption function features such a property.

Clearly, inverting the encryption function does not rely on the composite residuosity class problem, since this time the ciphertext is known to be an element of $\langle g\rangle$, but on a weaker instance. More formally,

Theorem 19. We call Partial Discrete Logarithm Problem the computational problem $P D L[n, g]$ defined as follows : given $w \in\langle g\rangle$, compute $\llbracket w \rrbracket_{g}$. Then Scheme 3 is one-way if and only if PDL $[n, g]$ is hard.

Theorem 20. We call Decisional Partial Discrete Logarithm Problem the decisional problem $D-P D L[n, g]$ defined as follows : given $w \in\left\langle g>\right.$ and $x \in \mathbb{Z}_{n}$, decide whether $\llbracket w \rrbracket_{g}=x$. Then Scheme 3 is semantically secure if and only if $D-P D L[n, g]$ is hard. 
The proofs are similar to those given in section 4. By opposition to the original class problems, these ones are not random-self-reducible over $g \in \mathcal{B}$ but over cyclic subgroups of $\mathcal{B}$, and present other interesting characteristics that we do not discuss here due to the lack of space. Obviously,

$$
\operatorname{PDL}[n, g] \Leftarrow \operatorname{Class}[n] \text { and } \operatorname{D-PDL}[n, g] \Leftarrow \mathrm{CR}[n]
$$

but equivalence can be reached when $g$ is of maximal order $n \lambda$ and $n$ the product of two safe primes. When $g \in \mathcal{B}_{\alpha}$ for some $\alpha<\lambda$ such that $|\alpha|=\Omega\left(|n|^{\epsilon}\right)$ for $\epsilon>0$, we conjecture that both $\mathrm{PDL}[n, g]$ and $\mathrm{D}-\mathrm{PDL}[n, g]$ are intractable.

In order to thwart Baby-Step Giant-Step attacks, we recommend the use of 160-bit prime numbers for $\alpha \mathrm{s}$ in practical use. This can be managed by an appropriate key generation. In this setting, the computational load of Scheme 3 is smaller than a RSA decryption with Chinese Remaindering for $|n| \geq 1280$. Next section provides tight evaluations and performance comparisons for all the encryption schemes presented in this paper.

\section{$7 \quad$ Efficiency and Implementation Aspects}

In this section, we briefly analyse the main practical aspects of computations required by our cryptosystems and provide various implementation strategies for increased performance.

Key Generation. The prime factors $p$ and $q$ must be generated according to the usual recommandations in order to make $n$ as hard to factor as possible. The fast variant (Scheme 3 ) requires additionally $\lambda=\operatorname{lcm}(p-1, q-1)$ to be a multiple of a 160-bit prime integer, which can be managed by usual DSA-prime generation or other similar techniques. The base $g$ can be chosen randomly among elements of order divisible by $n$, but note that the fast variant will require a specific treatment (typically raise an element of maximal order to the power $\lambda / \alpha$ ). The whole generation may be made easier by carrying out computations separately $\bmod p^{2}$ and $\bmod q^{2}$ and Chinese-remaindering $g \bmod p^{2}$ and $g \bmod q^{2}$ at the very end.

Encryption. Encryption requires a modular exponentiation of base $g$. The computation may be significantly accelerated by a judicious choice of $g$. As an illustrative example, taking $g=2$ or small numbers allows an immediate speed-up factor of $1 / 3$, provided the chosen value fulfills the requirement $g \in \mathcal{B}$ imposed by the setting. Optionally, $g$ could even be fixed to a constant value if the key generation process includes a specific adjustment. At the same time, pre-processing techniques for exponentiating a constant base can dramatically reduce the computational cost. The second computation $r^{n}$ or $g^{n r} \bmod n^{2}$ can also be computed in advance.

Decryption. Computing $\mathrm{L}(u)$ for $u \in \mathcal{S}_{n}$ may be achieved at a very low cost (only one multiplication modulo $2^{|n|}$ ) by precomputing $n^{-1} \bmod 2^{|n|}$. The constant parameter 


$$
\mathrm{L}\left(g^{\lambda} \bmod n^{2}\right)^{-1} \bmod n \text { or } \mathrm{L}\left(g^{\alpha} \bmod n^{2}\right)^{-1} \bmod n
$$

can also be precomputed once for all.

Decryption using Chinese-remaindering. The Chinese Remainder Theorem 6] can be used to efficiently reduce the decryption workload of the three cryptosystems. To see this, one has to employ the functions $\mathrm{L}_{p}$ and $\mathrm{L}_{q}$ defined over

$$
\mathcal{S}_{p}=\left\{x<p^{2} \mid x=1 \bmod p\right\} \quad \text { and } \quad \mathcal{S}_{q}=\left\{x<q^{2} \mid x=1 \bmod q\right\}
$$

by

$$
\mathrm{L}_{p}(x)=\frac{x-1}{p} \quad \text { and } \quad \mathrm{L}_{q}(x)=\frac{x-1}{q} .
$$

Decryption can therefore be made faster by separately computing the message $\bmod p$ and $\bmod q$ and recombining modular residues afterwards :

$$
\begin{aligned}
m_{p} & =\mathrm{L}_{p}\left(c^{p-1} \bmod p^{2}\right) h_{p} \bmod p \\
m_{q} & =\mathrm{L}_{q}\left(c^{q-1} \bmod q^{2}\right) h_{q} \bmod q \\
m & =\mathrm{CRT}\left(m_{p}, m_{q}\right) \bmod p q
\end{aligned}
$$

with precomputations

$$
\begin{aligned}
& h_{p}=\mathrm{L}_{p}\left(g^{p-1} \bmod p^{2}\right)^{-1} \bmod p \text { and } \\
& h_{q}=\mathrm{L}_{q}\left(g^{q-1} \bmod q^{2}\right)^{-1} \bmod q .
\end{aligned}
$$

where $p-1$ and $q-1$ have to be replaced by $\alpha$ in the fast variant.

Performance evaluations. For each $|n|=512, \cdots, 2048$, the modular multiplication of bitsize $|n|$ is taken as the unitary operation, we assume that the execution time of a modular multiplication is quadratic in the operand size and that modular squares are computed by the same routine. Chinese remaindering, as well as random number generation for probabilistic schemes, is considered to be negligible. The RSA public exponent is taken equal to $\mathrm{F}_{4}=2^{16}+1$. The parameter $g$ is set to 2 in our main scheme, as well as in the trapdoor permutation. Other parameters, secret exponents or messages are assumed to contain about the same number of ones and zeroes in their binary representation. 


\begin{tabular}{|l|c|c|c|c|c|}
\hline Schemes & Main Scheme & Permutation & Fast Variant & RSA & ElGamal \\
\hline One-wayness & Class $[n]$ & $\mathrm{RSA}[n, n]$ & $\mathrm{PDL}[n, g]$ & $\mathrm{RSA}\left[n, \mathrm{~F}_{4}\right]$ & $\mathrm{DH}[p]$ \\
Semantic Sec. & $\mathrm{CR}[n]$ & none & $\mathrm{D}-\mathrm{PDL}[n, g]$ & none & $\mathrm{D}-\mathrm{DH}[p]$ \\
\hline Plaintext size & $|n|$ & $2|n|$ & $|n|$ & $|n|$ & $|p|$ \\
Ciphertext size & $2|n|$ & $2|n|$ & $2|n|$ & $|n|$ & $2|p|$ \\
\hline
\end{tabular}

\begin{tabular}{|c|c|c|c|c|c|}
\hline Encryption & 5120 & 5120 & 4032 & $\mathbf{1 7}$ & 1536 \\
\hline$|n|,|p|=512$ & 7680 & 7680 & 5568 & $\mathbf{1 7}$ & 2304 \\
$|n|,|p|=768$ & 10240 & 10240 & 7104 & $\mathbf{1 7}$ & 3072 \\
$|n|,|p|=1024$ & 15360 & 1536 & 10176 & $\mathbf{1 7}$ & 4608 \\
$|n|,|p|=1536$ & 20480 & 20480 & 13248 & $\mathbf{1 7}$ & 6144 \\
$|n|,|p|=2048$ & & & & & \\
\hline
\end{tabular}

\begin{tabular}{|l|c|c|c|c|c|}
\hline Decryption & \multicolumn{1}{|c|}{} \\
\hline$|n|,|p|=512$ & 768 & 1088 & 480 & $\mathbf{1 9 2}$ & 768 \\
$|n|,|p|=768$ & 1152 & 1632 & 480 & $\mathbf{2 8 8}$ & 1152 \\
$|n|,|p|=1024$ & 1536 & 2176 & 480 & $\mathbf{3 8 4}$ & 1536 \\
$|n|,|p|=1536$ & 2304 & 3264 & $\mathbf{4 8 0}$ & 576 & 2304 \\
$|n|,|p|=2048$ & 3072 & 4352 & $\mathbf{4 8 0}$ & 768 & 3072 \\
\hline
\end{tabular}

These estimates are purely indicative, and do not result from an actual implementation. We did not include the potential pre-processing stages. Chinese remaindering is taken into account in cryptosystems that allow it i.e. all of them excepted ElGamal.

\section{Properties}

Before concluding, we would like to stress again the algebraic characteristics of our cryptosystems, especially those of Schemes 1 and 3.

Random-Self-Reducibility. This property actually concerns the underlying number-theoretic problems CR $[n]$ and Class $[n]$ and, to some extent, their weaker versions D-PDL $[n, g]$ and PDL $[n, g]$. Essentially, random-self-reducible problems are as hard on average as they are in the worst case : both RSA and the Discrete Log problems have this feature. Problems of that type are believed to yield good candidates for one-way functions [1].

Additive Homomorphic Properties. As already seen, the two encryption functions $m \mapsto g^{m} r^{n} \bmod n^{2}$ and $m \mapsto g^{m+n r} \bmod n^{2}$ are additively homomorphic on $\mathbb{Z}_{n}$. Practically, this leads to the following identities : 


$$
\begin{aligned}
& \forall m_{1}, m_{2} \in \mathbb{Z}_{n} \quad \text { and } \quad k \in \mathbb{N} \\
& \mathrm{D}\left(\mathrm{E}\left(m_{1}\right) \mathrm{E}\left(m_{2}\right) \bmod n^{2}\right)=m_{1}+m_{2} \bmod n \\
& \mathrm{D}\left(\mathrm{E}(m)^{k} \bmod n^{2}\right)=k m \bmod n \\
& \mathrm{D}\left(\mathrm{E}\left(m_{1}\right) g^{m_{2}} \bmod n^{2}\right)=m_{1}+m_{2} \bmod n \\
& \left.\begin{array}{l}
\mathrm{D}\left(\mathrm{E}\left(m_{1}\right)^{m_{2}} \bmod n^{2}\right) \\
\mathrm{D}\left(\mathrm{E}\left(m_{2}\right)^{m_{1}} \bmod n^{2}\right)
\end{array}\right\}=m_{1} m_{2} \bmod n .
\end{aligned}
$$

These properties are known to be particularly appreciated in the design of voting protocols, threshold cryptosystems, watermarking and secret sharing schemes, to quote a few. Server-aided polynomial evaluation (see [27]) is another potential field of application.

Self-Blinding. Any ciphertext can be publicly changed into another one without affecting the plaintext :

$$
\begin{gathered}
\forall m \in \mathbb{Z}_{n} \quad \text { and } \quad r \in \mathbb{N} \\
\mathrm{D}\left(\mathrm{E}(m) r^{n} \bmod n^{2}\right)=m \quad \text { or } \quad \mathrm{D}\left(\mathrm{E}(m) g^{n r} \bmod n^{2}\right)=m,
\end{gathered}
$$

depending on which cryptosystem is considered. Such a property has potential applications in a wide range of cryptographic settings.

\section{Further Research}

In this paper, we introduced a new number-theoretic problem and a related trapdoor mechanism based on the use of composite degree residues. We derived three new cryptosystems based on our technique, all of which are provably secure under adequate intractability assumptions.

Although we do not provide any proof of security against chosen ciphertext attacks, we believe that one could bring slight modifications to Schemes 1 and 3 to render them resistant against such attacks, at least in the random oracle model.

Another research topic resides in exploiting the homomorphic properties of our systems to design distributed cryptographic protocols (multi-signature, secret sharing, threshold cryptography, and so forth) or other cryptographically useful objects.

\section{Acknowledgments}

The author is especially grateful to David Pointcheval for his precious comments and contributions to this work. We also thank Jacques Stern and an anonymous 
referee for having (independently) proved that Class $[n] \Leftarrow \operatorname{RSA}[n, n]$. Finally, Dan Boneh, Jean-Sébastien Coron, Helena Handschuh and David Naccache are acknowledged for their helpful discussions and comments during the completion of this work.

\section{References}

1. D. Angluin and D. Lichtenstein, Provable Security of Cryptosystems: A Survey, Computer Science Department, Yale University, TR-288, 1983.

2. M. Bellare and P. Rogaway, Random Oracles are Practical : a Paradigm for Designing Efficient Protocols, In Proceedings of the First CCS, ACM Press, pp. 62-73, 1993.

3. J. C. Benaloh, Verifiable Secret-Ballot Elections, PhD Thesis, Yale University, 1988.

4. R. Cramer, R. Gennaro and B. Schoenmakers, A Secure And Optimally Efficient Multi-Authority Election Scheme, LNCS 1233, Proceedings of Eurocrypt'97, Springer-Verlag, pp. 103-118, 1997.

5. W. Diffie and M. Hellman, New Directions in Cryptography, IEEE Transaction on Information Theory, IT-22,6, pp. 644-654, 1995.

6. C. Ding, D. Pei and A. Salomaa, Chinese Remainder Theorem - Applications in Computing, Coding, Cryptography, World Scientific Publishing, 1996.

7. T. ElGamal, A Public-Key Cryptosystem an a Signature Scheme Based on Discrete Logarithms, IEEE Trans. on Information Theory, IT-31, pp. 469-472, 1985.

8. J. Feigenbaum, Locally Random Reductions in Interactive Complexity Theory, in Advances in Computational Complexity Theory, DIMACS Series on Discrete Mathematics and Theoretical Computer Science, vol. 13, American Mathematical Society, Providence, pp. 73-98, 1993.

9. S. Goldwasser and S. Micali, Probabilistic Encryption, JCSS Vol. 28 No 2, pp. 270-299, 1984.

10. K. Koyama, U. Maurer, T. Okamoto and S. Vanstone, New Public-Key Schemes based on Elliptic Curves over the ring Zn, LNCS 576, Proceedings of Crypto'91, Springer-Verlag, pp. 252-266, 1992.

11. T. Matsumoto and H. Imai, Public Quadratic Polynomial-Tuples for Efficient Signature-Verification and Message-Encryption, LNCS 330, Proceedings of Eurocrypt'88, Springer-Verlag, pp. 419-453, 1988.

12. U. Maurer and S. Wolf, On the Complexity of Breaking the Diffie-Hellman Protocol.

13. R. Merkle and M. Hellman, Hiding Information and Signatures in Trapdoor Knapsacks, IEEE Trans. on Information Theory, Vol. 24, pp. 525-530, 1978.

14. K. McCurley, A Key Distribution System Equivalent to Factoring, Journal of Cryptology, Vol. 1, pp. 95-105, 1988.

15. R. McEliece, A Public-Key Cryptosystem Based on Algebraic Coding Theory, DSN Progress Report 42-44, Jet Propulsion Laboratories, Pasadena, 1978.

16. D. Naccache and J. Stern, A New Public-Key Cryptosystem Based on Higher Residues, LNCS 1403, Advances in Cryptology, Proceedings of Eurocrypt'98, Springer-Verlag, pp. 308-318, 1998. 
17. D. Naccache and J. Stern, A New Public-Key Cryptosystem, LNCS 1233, Advances in Cryptology, Proceedings of Eurocrypt'97, Springer-Verlag, pp. 27-36, 1997.

18. P. Nguyen and J. Stern, Cryptanalysis of the Ajtai-Dwork Cryptosystem, LNCS 1462, Proceedings of Crypto'98, Springer-Verlag, pp. 223-242, 1998.

19. T. Okamoto and S. Uchiyama, A New Public-Key Cryptosystem as secure as Factoring, LNCS 1403, Advances in Cryptology, Proceedings of Eurocrypt'98, Springer-Verlag, pp. 308-318, 1998.

20. S. Park and D. Won, A Generalization of Public-Key Residue Cryptosystem, In Proceedings of 1993 Korean-Japan Joint Workshop on Information Security and Cryptology, pp. 202-206, 1993.

21. J. Patarin, The Oil and Vinegar Algorithm for Signatures, presented at the Dagstuhl Workshop on Cryptography, 1997.

22. J. Patarin and L. Goubin, Trapdoor One-Way Permutations and Multivariate Polynomials, LNCS 1334, Proceedings of ICICS'97, Springer-Verlag, pp. 356-368, 1997.

23. R. Peralta and E. Okamoto, Faster Factoring of Integers of a Special Form, IEICE, Trans. Fundamentals, E79-A, Vol. 4, pp. 489-493, 1996.

24. M. Rabin, Digital Signatures and Public-Key Encryptions as Intractable as Factorization, MIT Technical Report No 212, 1979.

25. R. Rivest, A. Shamir and L. Adleman, A Method for Obtaining Digital Signatures and Public-Key Cryptosystems, Communications of the ACM, Vol. 21, No 2, pp. 120-126, 1978.

26. A. Salomaa, Public-Key Cryptography, Springer-Verlag, 1990.

27. T. Sander and F. Tschudin, On Software Protection Via Function Hiding, Proceedings of Information Hiding Workshop'98, 1998.

28. S. Vanstone and R. Zuccherato, Elliptic Curve Cryptosystem Using Curves of Smooth Order Over the Ring $Z_{n}$, IEEE Trans. Inf. Theory, Vol. 43, No 4, July 1997.

29. S. Vaudenay, Cryptanalysis of the Chor-Rivest Cryptosystem, LNCS 1462, Proceedings of Crypto'98, Springer-Verlag, pp. 243-256, 1998.

30. H. Williams, Some Public-Key Crypto-Functions as Intractable as Factorization, LNCS 196, Proceedings of Crypto'84, Springer-Verlag, pp. 66-70, 1985. 